\title{
Effect of Acupressure on Constipation in Patients with Cancer
}

\author{
EunJin Lee* \\ Department of Nursing, Inha University, South Korea
}

Submission: November 01, 2017; Published: November 14, 2017

*Corresponding author: EunJin Lee, Associate professor, Department of Nursing, Inha University, Inharo 100, Namgu, Incheon, South Korea, Tel:+8232-860-8214; Email: eunjinlee@inha.ac.kr

\section{Introduction}

Patients with cancer often suffer from constipation. The prevalence of constipation in patients with cancer ranges from $42.7 \%$ to $87 \%$ [1,2]. A study of 206 patients with cancer reported that $74.3 \%$ took laxatives [2]. Americans spend \$ 725 million on laxatives each year [3]. Long term use of laxatives may have side effects such as bloating, allergic reaction, abdominal pain, metabolic disturbances, and hepatotoxicity [4]. Constipation decreases quality of life and is related to depressive symptoms $[5,6]$. Acupressure is the application of pressure or localized massage to specific sites on the body to manage symptoms such as pain or nausea [7]. Acupressure can be an alternative and cost-effective therapy for constipation which has fewer side effects than laxatives. The purpose of this study was to examine the effect of acupressure on constipation in cancer patients with constipation.

\section{Keywords}

\section{Acupressure; Constipation; Cancer}

\section{Methods}

A pilot study was conducted from February 1, 2010 through March 5, 2010 with eight cancer patients who were constipated. Prior to beginning the study, approval from the UK Markey Cancer Center IRB and the UK Medical IRB was obtained. Subjects were recruited from UK Markey Cancer Center chemotherapy outpatient department. The Constipation Assessment Scale (CAS) was used to measure constipation severity [8]. The experimental group was instructed to perform 5 minute acupressure at SP 14 every day for 7 days. The placebo group was instructed to perform 5 minute acupressure daily at PC 6 for 7 days. Left SP 14 is located at 1.3 cun below and 4 cun left lateral to the umbilicus.

\section{Results}

The basic characteristics of 8 patients were a mean age $62.5( \pm 7.58)$ years, $100 \%$ female, and $62 \%$ unemployed status. Their mean number of months since cancer diagnosis was
$13.1( \pm 19.9)$ months; the majority had stage three cancer and all were presently receiving chemotherapy. The characteristics of constipation were: mean onset of constipation were 10.3 weeks $( \pm 16.11)$ not including one outlier-- a patient who had experienced constipation for 20 years; $86 \%$ used laxatives, stool softeners, or enemas and averaged $5.5( \pm 3.8)$ bowel movements per week. Three out of five patients in the experimental group reported improvement of constipation in terms of either the frequency of bowel movements or the CAS scores. Two out of three patients in the placebo group reported that acupressure did not help constipation even though they had improvement of constipation in the frequency of bowel movements and laxatives use.

\section{Case Description}

Ms. Jones, a 59-year-old female, had multiple myeloma for 60 months with co-morbidity of hypertension and hyperlipidemia. She had constipation for 2 weeks since she started the new chemotherapy (Cafilzomib). She did not take any laxatives, suppositories, stool softeners, or enemas for constipation. She drank prune juice for constipation, more than 5 cups of fluid, and ate 1 cup of fruits and vegetables for the 24 hours, and had not exercised for the past week. She had taken six Aleve tablets for the past week. She wanted to learn acupressure because she did not like taking medication for constipation. She performed acupressure for 5 minutes, a total of five times for one week. Bowel movements increased from two to five times per week. She did not take any medication for constipation for the past week. Her score on the Constipation Assessment Scale did not improve. She reported that performing acupressure was easy and it worked. She rated the effect of acupressure on constipation as 4 out of 5 (very helpful). She did not have any side effects.

\section{Discussion}

The results of this study are consistent with previous studies. Auricular acupressure reduced constipation compared 
to the control group in 52 patients with cancer ( $<$ <. 001) [9]. Acupressure at RN12, LI11, and ST25 for 10 days improved constipation compared to the control group in 78 patients with psychiatric diseases $(p=0.0003)$ [10]. There are a few studies that examined effect of acupressure on constipation. Therefore further studies are needed to examine the effect of acupressure on constipation using a randomized controlled trial design.

\section{Conclusion}

Acupressure at SP 14 for 5 minutes, daily for 1 week was effective in improving constipation in three out of five cancer patients with constipation. Further study is needed to examine the effect of acupressure using a revised experimental protocol. The revised protocol will use two acupressure points at CV4 and SP14 for 10 minutes with a lying down position.

\section{Funding}

This work was supported by an INHA University Research Grant (52704).

\section{Conflict Of Interest}

None

\section{References}

1. Sykes NP (1998) The relationship between opioid use and laxative use in terminally ill cancer patients. Palliat Med 12(5): 375-382.

2. Wirz S, Klaschik E (2005) Management of constipation in palliative care patients undergoing opioid therapy: is polyethylene glycol an option? Am J Hosp Palliat Care 22(5): 375-381.

3. (2007) Constipation. National Institute of Diabetes and Digestive and Kidney Diseases.

4. Xing JH, Soffer EE (2001) Adverse effects of laxatives. Dis Colon Rectum 44(8): 1201-1209.

5. Bengtsson M, Ohlsson B (2005) Psychological well-being and symptoms in women with chronic constipation treated with sodium picosulphate. Gastroenterol Nurs 28(1): 3-12.

6. Thomas JR, Cooney GA, Slatkin NE (2008) Palliative care and pain: new strategies for managing opioid bowel dysfunction. J Palliat Med 11 (Suppl 1): S1-19; quiz S21-12.

7. (2010) NCI Dictionary of cancer terms. National Cancer Institute. Acupressure.

8. Mcmillan SC, Williams FA (1989) Validity and reliability of the constipation assessment scale. Cancer Nurs 12(3): 183-188.

9. Shin J, Park H (2016) Effects of auricular acupressure on constipation in patients with breast cancer receiving chemotherapy: A randomized control trial. West J Nurs Res 193945916680362.

10. Wong WK, Chien WT, Lee WM (2015) Self-administered acupressure for treating adult psychiatric patients with constipation: a randomized controlled trial. Chin Med 10: 32

\section{Your next submission with Juniper Publishers will reach you the below assets}

- Quality Editorial service

- Swift Peer Review

- Reprints availability

- E-prints Service

- Manuscript Podcast for convenient understanding

- Global attainment for your research

- Manuscript accessibility in different formats

( Pdf, E-pub, Full Text, Audio)

- Unceasing customer service

Track the below URL for one-step submission https://juniperpublishers.com/online-submission.php 